\title{
Pattern of dopaminergic loss in the striatum of humans with MPTP induced parkinsonism
}

Barry J Snow, Francois J G Vingerhoets, J William Langston, James W Tetrud, Vesna Sossi, Donald B Calne
The

Neurodegenerative

Disorders Centre,

University of British

Columbia, Vancouver,

BC, Canada

B J Snow

F J G Vingerhoets

D B Calne

University of British Columbia, TRIUMF, Vancouver, BC,

Canada

V Sossi

The Parkinson's Institute, 1170 Morse Avenue, Sunnyvale,

California, USA

$\mathrm{J}$ W Langston

J W Tetrud

Correspondence to: Dr Barry J Snow,

Department of Neurology, Auckland Hospital, Private Bag 92024, Auckland, New Zealand. Telephone 006425 314439; fax 00649307

4924; email:

bsnow@ahsl.co.nz

Received 28 July 1998 and in revised form

8 June 1999

Accepted 16 August 1999

\begin{abstract}
Objectives-To examine the distribution of striatal dopaminergic function in humans with parkinsonism induced by 1-methyl-4phenyl-1,2,3,6-tetrahydropyridine (MPTP) to determine if there is a caudateputamen gradient as is seen in idiopathic Parkinson's disease.

Methods-We scanned nine humans exposed to MPTP with parkinsonism ranging from minimal to severe using $\left[{ }^{18} \mathrm{~F}\right]$ fluorodopa (FD) and high resolution PET. The results were compared with those of 10 patients with Parkinson's disease and six normal subjects.

Results-In the MPTP group there was an equal degree of reduction of dopaminergic function in the caudate and putamen. This was different from the greater putaminal than caudate loss in Parkinson's disease $(\mathrm{p}<0.001)$.

Conclusions-Parkinson's disease is not caused by transient exposure to MPTP.

(F Neurol Neurosurg Psychiatry 2000;68:313-316)

Keywords: 1-Methyl-4-phenyl-1,2,3,6-tetrahydropyridine (MPTP); Parkinson's disease; positron emission tomography; fluorodopa
\end{abstract}

1-Methyl-4-phenyl-1,2,3,6-tetrahydropyridine (MPTP) produces parkinsonism in both humans and animals. ${ }^{12}$ Clinically, the syndrome may be almost indistinguishable with bradykinesia, rigidity, impaired postural reflexes, sometimes tremor, and a neuropsychological deficit similar to Parkinson's disease. ${ }^{3}$ In some humans with parkinsonism induced by MPTP, the clinical deficit and the dopaminergic deficit as disclosed by PET, is also progressive over time. ${ }^{4}$ Aged monkeys treated chronically with MPTP have been reported to have inclusions in nigral dopaminergic neurons that are reminiscent of Lewy bodies. ${ }^{5}$

A characteristic feature of Parkinson's disease is the striatal gradient of loss of indices of striatal dopaminergic function, with the greatest deficit being in the putamen. ${ }^{6}$ Experiments with MPTP in non-human primates have found different patterns of striatal dopamine deficit. ${ }^{7-13}$ Some studies have found the loss of caudate dopamine to be equal or greater than the dopamine loss from the putamen, and it has been suggested that this finding indicates that a substance similar to MPTP is unlikely to be the underlying cause of Parkinson's disease. ${ }^{8}$ By contrast with those findings, other workers have produced a caudate putamen gradient similar to that in Parkinson's disease using low doses or chronic administration of MPTP to primates. ${ }^{1012} 13$ There is a similar variation in findings of the patterns of cell and dopamine loss in the substantia nigra. ${ }^{514} 15$ The postmortem distribution of the striatal dopaminergic deficit in human MPTP parkinsonism has not yet been described.

The regional variation of striatal dopamine loss in Parkinson's disease is clearly shown with $\left[{ }^{18} \mathrm{~F}\right]$-fluorodopa (FD) PET using high resolution tomographs. ${ }^{16}{ }^{17}$ Our previous reports of FD PET in human parkinsonism induced by MPTP described scans performed on a PETT VI scanner with a resolution insufficient to discriminate caudate from putamen. ${ }^{4}{ }^{18}$ We now present the results of FD PET of human MPTP parkinsonism on a high resolution scanner. We used these data to examine the pattern of the striatal dopaminergic loss in MPTP parkinsonism.

\section{Methods}

All assessments were performed at the University of British Columbia. Nine subjects were studied about 10 years after having been exposed to MPTP. Eight had mild parkinsonism (mean age 39.4, range 30-55, men/women $4 / 4$, mean modified Columbia score $\left(\mathrm{MCS}^{19}\right)$ while off medication 6.4, range 2-16). Detailed descriptions of their clinical status and total striatal PET results have been presented recently. ${ }^{4}$ In addition, a severely affected woman aged 34 years with Hoehn and $\mathrm{Yahr}^{20}$ stage V parkinsonism and an MCS of 70 was scanned.

Two control groups were scanned. The first consisted of 10 patients with Parkinson's disease (mean age 53.6, range 33-74; four men, six women; mean MCS 22.9, range 4-44). The second consisted of six normal subjects (mean age 66.0, range 52-80; twomen, four women).

This study was performed on patients who were being rescanned on a PETT VI scanner as part of our longitudinal study reported previously. ${ }^{4}$ That scanner does not have sufficient resolution to examine the putamen/ caudate ratio. We therefore devised a protocol where we followed the PETT VI scan with a further scan on a high resolution scanner. By 2 hours after injection ${ }^{18} \mathrm{~F}$ has decayed for more than one half life. To deal with the reduced signal we scanned on an ECAT 953-31B scanner in $3 \mathrm{D}$ mode. ${ }^{21}$ This increases the number of recorded true coincidences by a factor of six, which more than compensates for the effect of radioactive decay. The ECAT scanner has a resolution of $5.6 \times 5.6 \times 5 \mathrm{~mm}$, full width at half 


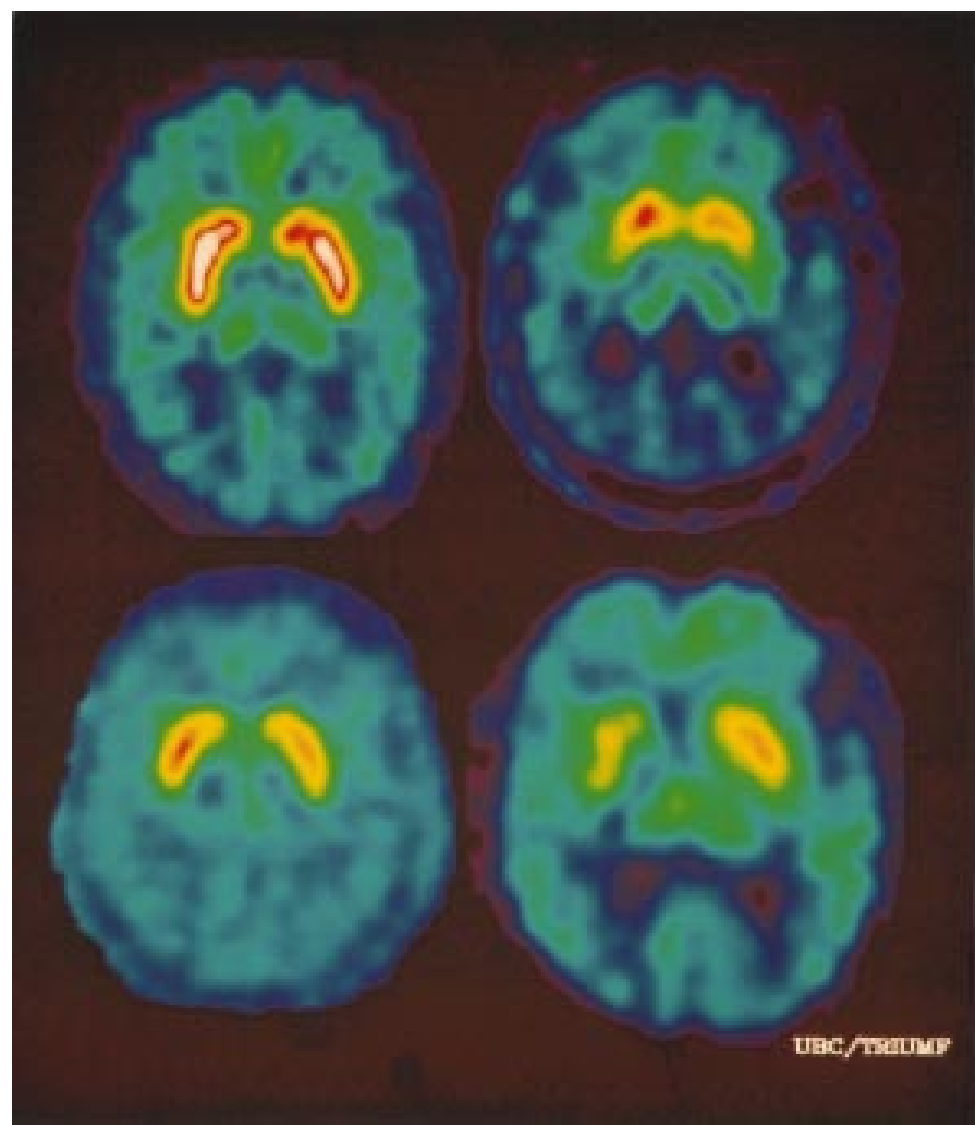

Figure 1 Representative FD PET images of a normal subject (top left), patient with Parkinson's disease (top right), a mildly affected MPTP exposed subject (bottom left), and the most severely affected MPTP exposed subject (bottom right). The greater degree of putaminal loss is seen in the patient with Parkinson's disease, whereas the MPTP exposed subjects have homogeneous losses of striatal signal. The colour scale represents radioactivity concentration.

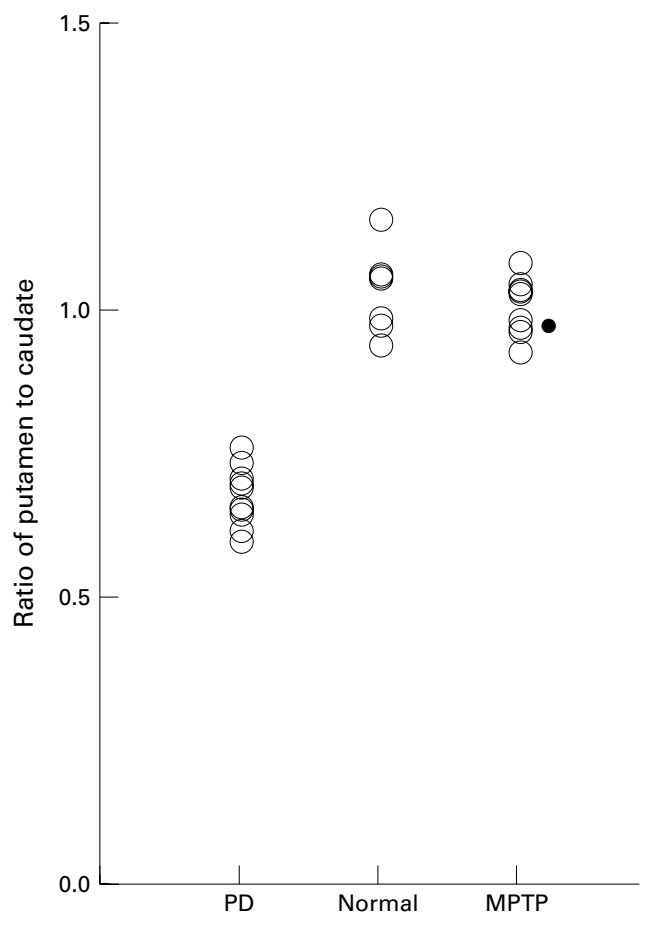

Figure 2 Scatterplots of the putamen/caudate ratio for the subject groups; $\bullet$ indicates the most severely affected MPTP exposed subject. The MPTP exposed group was significantly different from the control subjects ( $p=1.9 E$ 10) but no different from the normal group.

the five slices where the striata were best seen. The mean bilateral caudate and putamen values were then calculated and expressed as a ratio of putamen/caudate.

\section{Results}

All results are from the ECAT scanner. The images from the subjects with Parkinson's disease showed marked putaminal loss of signal with a putamen/caudate ratio of 0.68 (SD 0.053) (figs 1 and 2). By contrast, the normal subjects had a homogeneous distribution of striatal radioactivity with a putamen/caudate ratio of 1.02 (SD 0.053). The MPTP parkinsonian subjects had varying degrees of loss of striatal signal with the most severe depletion in the clinically most affected patient (fig 1). The loss was always uniform; the putamen/caudate ratio was 1.01 (SD 0.050). The severely affected patient had a ratio of 0.98 . The putamen/caudate ratios in the Parkinson's disease group were significantly different from the MPTP exposed $(\mathrm{p}=1.9 \mathrm{E} 10$, unpaired, two tailed $t$ test on the log of the ratios) and normal groups $(p=2.03 \mathrm{E} 8)$. There was no difference between the putamen/caudate ratios of the MPTP exposed and normal groups. In the MPTP exposed group, there was no significant correlation between the putamen/caudate ratio and the overall severity of the dopaminergic lesion, the MCS, or with the age of the subject. The asymmetry of the ratios expressed as a percentage of the subject's uptake varied from $0-14 \%$ (mean 5\%) in the MPTP exposed group. This was significantly less than the asymmetry in the Parkinson's disease group, which varied from $2 \%-23 \%$ (mean $12 \%$, $\mathrm{p}=0.016)$. 
The mean age and mean Columbia scores of the Parkinson's disease group were greater than those of the MPTP exposed group. Within the Parkinson's disease group, however, there was no correlation between the putamen/caudate ratios and either the age or the Columbia score.

\section{Discussion}

We have shown that in human MPTP induced parkinsonism there is an equal loss of dopaminergic function from the caudate and putamen. This contrasts with the greater putaminal loss seen in Parkinson's disease both in this study and other reports. ${ }^{6} 1617$ Other forms of parkinsonism are associated with a uniform loss of striatal signal on FD PET. In particular, in progressive supranuclear palsy there is equal loss of caudate and putaminal signal, and progressive supranuclear palsy may be distinguished from Parkinson's disease with a high degree of certainty on the basis of the pattern of striatal FD uptake. ${ }^{17}{ }^{25}$ Cortical basal ganglionic degeneration and post-traumatic parkinsonism also have a uniform loss of striatal signal in PET studies. ${ }^{26}{ }^{27}$ Multiple system atrophy has a variable pattern with both uniform loss and greater putaminal loss found with PET and pathological studies. ${ }^{25} 28$

In non-human primates, acute administration of high doses of MPTP produces uniform striatal dopaminergic loss. ${ }^{12}$ By contrast, several studies have demonstrated that either a single low dose (for example, $1.5 \mathrm{mg} / \mathrm{kg}$ subcutaneously in the squirrel monkey) or chronic low doses of MPTP consistently produce a greater depletion of dopaminergic markers in the putamen than the caudate, ${ }^{1329}$ a pattern similar to that found in Parkinson's disease. Such a pattern was not seen in any of the subjects with MPTP induced parkinsonism in this study. Furthermore, the putamen/caudate ratio did not correlate with either the overall severity of the striatal dopaminergic lesion or the clinical deficit. Two of our subjects reported that they injected drugs that were likely to contain MPTP over 3 to 4 months, a dosing schedule similar to that administered to the monkeys, yet PET demonstrated equal caudate and putaminal loss. It is possible that one or more of the injections of MPTP administered by these subjects was a high enough dose to cause a uniform caudate and putamen dopaminergic depletion. We also found that the MPTP exposed subjects had striatal dopaminergic function that was significantly more symmetric than the subjects with Parkinson's disease. Parkinson's disease typically presents asymmetrically, and this is reflected in the striatal fluorodopa uptake.

It might be argued that all of our patients were exposed to the equivalent of the high doses of MPTP that produce an equal loss of caudate and putamen dopaminergic function in animal studies. However, if this were the case, we might have expected the subjects to manifest clear cut signs of parkinsonism, yet three of our subjects were initially clinically normal and gave no history of transient parkinsonism. None the less, a feature of the animal work with MPTP is the variation in degree of dopaminergic deficit that develops in response to similar doses of the toxin. It therefore remains possible that humans might develop a striatal dopaminergic deficit with a distribution similar to Parkinson's disease if they were exposed to MPTP under different circumstances.

A further factor that needs to be considered is the effect of the subject's age on the pattern of striatal dopaminergic loss after exposure to MPTP. The mean age of our subjects was younger than the Parkinson's disease group, and it could be argued that the projection to the caudate is more vulnerable in younger subjects. Against this argument was the lack of any correlation between age and the putamen/ caudate ratio in the MPTP exposed group and the fact that there was an overlap in the ages of the MPTP exposed and Parkinson's disease groups.

It is not known why there is a differential vulnerability of nigral neurons to the pathogenic process that causes Parkinson's disease or to MPTP in some non-human primate experiments. ${ }^{30}$ There are regional differences within the nigra including variations in the concentrations of neuromelanin, calbindin, and fibroblast growth factor. ${ }^{31-35}$ There are also differences between the caudate and putamen in the concentrations of dopamine reuptake sites in the striatal nerve terminals of nigral neurons. ${ }^{36}$ The relevance of these findings to the neuronal loss in Parkinson's disease is not clear. $^{37}$

In conclusion, the subjects of our study have a pattern of striatal dopaminergic function that differentiates them from subjects with Parkinson's disease. This finding indicates that Parkinson's disease is not caused by transient MPTP exposure.

We thank the UBC/TRIUMF PET group, who helped with the PET scanning. This study was supported by the Medical Research Council of Canada, the National Parkinson Foundation, the Parkinson Foundation of Canada, the Movement Disorder Institute, Vancouver, and the Parkinson's Institute, Sunnyvale California.

1 Langston JW, Ballard PA, Tetrud JW, et al. Chronic parkinsonism in humans due to a product of meperidine-analog synthesis. Science 1983;219:979-80.

2 Burns RS, Chiueh CC, Markey SP, et al. A primate model of parkinsonism: selective destruction of dopaminergic neurons in the pars compacta of the substantia nigra by N-methyl-4phenyl-1,2,3,6-tetrahydropyridine. Proc Natl Acad Sci USA 1983;80:4546-50.

3 Stern Y, Tetrud JW, Martin WRW, et al. Cognitive change following MPTP exposure. Neurology 1990;40:261-4.

4 Vingerhoets FJG, Snow BJ, Tetrud JW, et al. Positron emission tomography evidence for progression of human MPTP-induced dopaminergic lesions. Ann Neurol 1994; 36:765-70.

5 Forno LS, DeLanney LE, Irwin I, et al. Similarities and differences between MPTP-induced parkinsonsim and Parkinson's disease. Neuropathologic considerations. $A d v$ Neurol 1993;60:600-8.

6 Kish SJ, Shannak K, Hornykiewicz O. Uneven pattern of dopamine loss in the striatum of patients with idiopathic Parkinson's disease. N Engl F Med 1988;318:876-80.

7 Elsworth JD, Deutch AY, Redmond DE, et al. Symptomatic and asymptomatic 1-methyl-4-phenyl-1,2,3,6-tetrahydropyridine-treated primates: biochemical changes in striatal regions. Neurosci 1989;33:323-31.

8 Pifl C, Schingnitz G, Hornykiewicz O. The neurotoxin MPTP does not reproduce in the resus monkey the interregional pattern of striatal dopamine loss typical of internan idical of 92:228-33.

9 Alexander GM, Schwartzman RJ, Brainard L, et al. Changes in brain catecholamines and dopamine uptake sites at different stages of MPTP parkinsonism in monkeys. Brain different stages of $M$
Res 1992;588:261-9. 
10 Hantraye P, Varastet M, Peschanski M, et al. Stable parkinsonian syndrome and uneven loss of striatal dopamine
fibres following chronic MPTP administration in baboons. Neurosci 1993;53:169-78.

11 Pifl C, Schingnitz G, Hornykiewicz O. Effect of 1-methyl-4phenyl-1,2,3,6-tetrahydropyridine on the regional distribution of brain monoamines in the rhesus monkey. Neurosci 1991;44:591-605.

12 Perez-Otano I, Oset C, Luquin MR, et al. MPTP-induced parkinsonism in primates: pattern of striatal dopamine loss following acute and chronic administration. Neurosci Let 1994;175:121-5.

13 Moratalla R, Quinn B, DeLanney LE, et al. Differential vulnerability of primate caudate-putamen and striosomematrix dopamine systems to the neurotoxic effects of 1-methyl-4-phenyl-1,2,3,6-tetrahydropyridine. Proc Nat Acad Sci USA 1992;89:3859-63.

14 German DC, Dubach M, Askari S, et al. 1-Methyl-4phenyl-1,2,3,6-tetrahydropyridine-induced parkinsonian phenyl-1,2,3,6-tetrahydropyridine-induced parkinsonian syndrome in Macaca fascicularis: which midbrain
dopaminergic neurons are lost? Neurosci $1988 ; 24: 161-74$

15 Elsworth JD, Deutch AY, Redmond DE, Jr, et al. MPTP-induced parkinsonism: relative changes in dopamine concentration in subregions of substantia nigra, ventral tegmental area and retrorubral field of symptomatic and asymptomatic vervet monkeys. Brain Res 1990;513 $320-4$

16 Garnett ES, Nahmias C, Firnau G. Central dopaminergic pathways in hemiparkinsonism examined by positron emission tomography. Can f Neurol Sci 1984;11:174-9.

17 Brooks DJ, Ibanez V, Sawle GV, et al. Differing patterns of striatal[18F]-dopa uptake in Parkinson's disease, multiple system atrophy, and progressive supranuclear palsy. $A n n$ Neurol 1990;28:547-55.

18 Calne DB, Langston JW, Martin WRW, et al. Positron emission tomography after MPTP: observations relating to the cause of Parkinson's disease. Nature 1985;317:246-8.

19 Duvoisin RC. The evaluation of extrapyramidal disease. In: de Ajuriaguerra J, Gauthier G. Monoamines noyaux gris cende Ajuriaguerra J, Gauthier G. Monoamines noyaux gris centraux et syndrome

20 Hoehn MM, Yahr MD. Parkinsonism: onset, progression and mortality. Neurology 1967;5:427-42.

21 Kinahan PE, Rogers JG. Analytic 3D image reconstruction using all detected events. IEEE Transactions on Nuclear Science 1989;36:964-8.

22 Cherry SR, Dahlbom M, Hoffman EJ. 3D PET using a conventional multislice tomograph. $\mathcal{F}$ Comput Assist Tomogr 1991;15:655-68.

23 Bailey DL, Meikle SR. A convolution-subtraction scatter correction method for 3D PET. Phys Med Biol 1994;39: 411-24.
24 Vingerhoets FJG, Snow BJ, Schulzer M, et al. Reproducibility of fluorine-18-6-fluorodopa positron emission tomgraphy in normal human subjects. 7 Nucl Med 1994;35: 8-24.

25 Burn DJ, Sawle GV, Brooks DJ. Differential diagnosis of Parkinson's disease, multiple system atrophy, and SteeleRichardson-Olszewski syndrome: discriminant analysis of striatal $\left[{ }^{18} \mathrm{~F}\right]$-dopa PET data. $\mathcal{F}$ Neurol Neurosurg Psychiatry 1994;57:278-4.

26 Sawle GV, Brooks DJ, Marsden CD, et al. Corticobasal degeneration. Brain 1991;114:541-56.

27 Turjanski N, Lees AJ, Brooks DJ. Dopaminergic function in patients with posttraumatic parkinsonism: an 18F-dopa patients with posttraumatic parkinso
PET study. Neurology 1997;49:183-9.

28 Spokes EGS, Bannister R, Oppenheimer DR. Multiple system atrophy with autonomic failure. $\mathcal{F}$ Neurol Sci 1979;43: $59-62$.

29 Irwin I, DeLanney LE, Forno LS, et al. The evolution of nigrostriatal neurochemical changes in the MPTP-treated squirrel monkey. Brain Res 1990;531:242-52.

30 Hirsch EC. Why are nigral catecholaminergic neurons more vulnerable than other cells in Parkinson's disease? Ann Neurol 1992;32:S88-93.

31 Hirsch E, Graybiel AM, Agid Y. Melanized dopaminergic neurons are differentially susceptable to degeneration in Parkinson's disease. Nature 1988;334:345-8.

32 Hirsch EC, Mouatt A, Thomasset M, et al. Expression of calbindin D28-like immunoreactivity in catecholaminergic cell groups of the human midbrain: normal distribution and distribution in Parkinson's disease. Neurodegeneration 992;1:83-93.

33 Gibb WRG, Lees AJ. Anatomy, pigmentation, ventral and dorsal subpopulations of the substantia nigra, and differential cell death in Parkinson's disease. 7 Neurol Neurosurg Psychiatry 1991;54:388-96.

34 Yamada T, McGeer PL, Baimbridge KG, et al. Relative sparing in Parkinson's disease of substantia nigra dopamine neurons containing calbindin-D28K. Brain Res 1990;525: 303-7.

35 German DC, Manaye K, Smith WK, et al. Midbrain dopaminergic cell loss in Parkinson's disease: computer visualization. Ann Neurol 1989;26:507-14.

36 Marshall JF, Navarrete RJ. Contrasting tissue factors predict heterogeneous striatal dopamine neurotoxicity after MPTP or methamphetamine treatment. Brain Res 1990;534:34851.

37 Gibb WR. Melanin, tyrosine hydroxylase, calbindin and substance $\mathrm{P}$ in the human midbrain and substantia nigra in relation to nigrostriatal projections and differential neuronal susceptibility in Parkinson's disease. Brain Res 1992; 581:283-91. 